\title{
Los usos escritos y orales del ayöök y del español en la representación de saberes comunitarios en una escuela indígena de Oaxaca, México
}

\author{
Oral and written uses of Ayöök and Spanish to represent \\ community knowledge in an indigenous school in Oaxaca, \\ Mexico
}

\author{
Julieta Briseño-Roa ${ }^{1}$ \\ Elsie Rockwell Richmond²
}

DOI: http://dx.doi.org/10.20435/tellus.vi43.701

\begin{abstract}
Resumo: La escritura de lenguas indígenas en contextos escolares en México no se puede comprender sin situar cada caso en su contexto socio-histórico. En este artículo, se analizan las representaciones escritas y gráficas de saberes hechas por jóvenes de 12 a 15 años que asisten a una secundaria con un modelo educativo de educación público alternativo en la región mixe de Oaxaca en México. A partir de material etnográfico resultado de más de un año de trabajo de campo, se presentan ejemplos de material construido por los estudiantes que muestran reflexiones sobre la lengua ayöök, así como las traducciones entre la lengua indígena y el español dentro de prácticas escolares. El trabajo tiene la intención de aportar a la discusión sobre el uso de lenguas indígenas y español sin detrimento una de otra en contextos educativos.
\end{abstract}

Palavras-chave: Educación indígena; escritura de lenguas indígenas; bilingüismo; etnografía; traducción.

\begin{abstract}
It is not possible to understand writing in indigenous languages in school contexts in Mexico without taking into account each particular socio-historic context. In this article, we analyze both graphic and written representations of knowledge produced by students between 12 to 15 years old who attend a public secondary school with an alternative education educational model in the Mixe region of Oaxaca, in Mexico. Through ethnographic data collected during more than a year of fieldwork, we show examples of the materials produced by the students and their reflections on their Ayöök
\end{abstract}

1 Centro de Investigaciones y Estudios Superiores en Antropología Social (CIESAS), Ciudad de México, México.

2 Centro de Investigaciones y Estudios Avanzados (Cinvestav). 
language, as well as translations from their native language to Spanish and back during school activities. Our research contributes to the discussion on how to use both indigenous languages and Spanish in classroom activities, without subordinating either one to the other.

Keywords: Indigenous education; writing indigenous languages; bilingualism; ethnography; translation.

\section{INTRODUÇÃO}

Examinar la situación del uso de las lenguas indígenas en contextos escolares y comunitarios en México no es tarea sencilla. Existe una gran diversidad de historias, condiciones y proyectos que desafían cualquier esquema de clasificación. La diferencia de ámbitos de uso de las lenguas originarias, tanto en lo oral como por escrito, en comunidades incluso cercanas entre sí, obliga a un análisis cuidadoso de cada caso, para comprender los sentidos y las formas que adquiere el uso de las lenguas indígenas dentro y fuera de las aulas.

En este artículo, se considera el caso de una escuela secundaria (en México se denomina secundaria a los tres últimos años de enseñanza fundamental 7으, 8ㅇ y 9ㅇ) en una comunidad indígena con una historia particular y con un modelo alternativo, pero revela procesos lingüísticos más generales. Cómo en todo el continente, se trata de procesos insertos en contextos de colonialidad (QUIJANO, 2014), marcados por dominación, pero también por profundas corrientes de resistencia cultural.

En México existe variabilidad en cuanto a la vitalidad de las lenguas originarias en las comunidades, es decir, en el uso activo que les dan las generaciones más jóvenes, lo cual incide en el riesgo de desplazamiento por el español (CIFUENTES; MOCTEZUMA, 2006). Si bien varias de las lenguas más importantes del país contaban con formas propias de escritura prehispánicas y fueron escritas con alfabeto latino durante el periodo colonial, estas tradiciones se perdieron paulatinamente. No obstante, ciertas representaciones gráficas del saber indígena, como el registro del territorio y de elementos históricos, sobrevivieron con fuerza en algunas regiones, junto con una transmisión oral intergeneracional de saberes propios en las lenguas. Sobre la escritura en contextos poscoloniales, Collins y Blot (2003) argumentan que la relación entre poder, lengua e identidad es compleja, por lo que no se trata de solo una imposición de sistemas de escritura. En esta relación los pueblos indígenas han encontrado espacios para sus propias voces dentro de 
prácticas letradas tanto tradicionales y modernas (COLLINS; BLOT, 2003). Estos espacios incluyen usos tan diversos como la divulgación oral o escrita de textos religiosos o cívicos en las lenguas indígenas y la emergencia de cantautores indígenas que producen en sus propias lenguas en la actualidad. Por otra parte, existen diversas formas de apropiación por parte de los pueblos del español escrito que se fueron construyendo en situación de opresión y dominación (PELLICER, 2010).

La educación escolar básica o fundamental es uniforme para toda la población y está marcada por la subordinación de las lenguas indígenas al español. Incluso en la enseñanza intercultural bilingüe, política oficial, la tendencia es "encuadrar" elementos de la lengua local (frases y léxico) como tema curricular dentro de un uso corriente del español como medio de enseñanza, es decir, como "lengua matriz" (MEEK; MESSING, 2007). No obstante, existe una considerable variación, que depende del grado de uso de la lengua por las generaciones jóvenes y de la disposición del maestro/a en turno. En el caso que analizamos se observan las posibilidades de resistir esa subordinación, pues los estudiantes son hablantes y trabajan por proyectos que implican investigar temas con las personas mayores de su comunidad.

Si bien México cuenta con décadas de educación pública para los pueblos indígenas impartida por maestros hablantes de las lenguas, ello no siempre ha significado que la enseñanza en las aulas se llevara a cabo usando las variantes conocidas por los alumnos. Los obstáculos son enormes, en México como en muchas partes (MCCARTY, 2003). Incluyen la asignación de maestros hablantes de variantes distintas a las de sus alumnos, una formación docente que privilegia el español y coarta el uso de las lenguas, y la carencia de materiales impresos en las lenguas. Sobre todo, el constante problema de las decisiones en torno a la estandarización ortográfica de las lenguas indígenas ha marcado esa historia. Este último punto ha llevado a cierto estancamiento, pues compiten diferentes propuestas, y los hablantes de cada variante reclaman su propio alfabeto, lo cual tiende a fragmentar los esfuerzos de producción de materiales escritos. De hecho, el proceso paraliza el uso cotidiano de la escritura de las lenguas, dado que los hablantes dicen no saber escribir "bien" su lengua, aunque se ha mostrado que cualquier niño alfabetizado en español también encuentra maneras de representar su propia lengua seleccionando combinaciones de grafías de manera espontánea (PELLICER, 2004)

A pesar de ello, hay larga historia de producción de materiales en las lenguas durante el último siglo, como anteriormente. Como en muchas partes, el Instituto 
Lingüístico de Verano (SIL por sus siglas en inglés), produjo a inicios del siglo veinte cartillas para las escuelas oficiales, cartillas de alfabetización inicial y cuentos en varias lenguas. A partir de la década de 1980, grupos de maestros bilingües con apoyo de la Secretaría de Educación Pública (SEP) empezaron a redactar manuales para el aprendizaje inicial de la escritura de las lenguas, y en la década de 1990 este proyecto se extendió a los grados superiores de la primaria. Al mismo tiempo, se abrieron algunas Escuelas Normales ${ }^{3}$ y Universidades Interculturales que hicieron diferentes esfuerzos para difundir las lenguas en forma escrita. A partir de su creación en 2003, el Instituto Nacional de Lenguas Indígenas (INALI) identificó más de 364 variantes de las 68 lenguas reconocidas (INALI, 2008), lo cual reforzó la postura que hacer libros para cada una sería una tarea inabarcable.

Paralelo a estos esfuerzos oficiales, sin embargo, surgieron en diversas regiones proyectos locales que hicieron uso efectivo de la escritura de las lenguas indígenas. Por ejemplo, algunas escuelas de la Coalición de Maestros y Promotores Indígenas de Oaxaca (CMPIO) emprendieron la práctica de fabricar a mano álbumes para niños, de gran formato, con textos en las lenguas (MEYER, 2010). En la región purépecha, los docentes de dos comunidades han trabajado durante dos décadas para producir material escrito para todas las asignaturas de los programas, y ha instalado el uso oral de la lengua de manera cotidiana en las aulas (HAMEL; BALTAZAR; MARTÍNEZ, 2018). En otros casos, los esfuerzos de revitalización se han emprendido fuera del sistema escolar como el caso de náhuatl que ha documentado sistemáticamente Flores Farfán (2020). Incluso la proliferación de los celulares se ha convertido en un medio para incentivar la escritura de mensajes en las lenguas (DE LEÓN, 2018). En este contexto, el presente artículo pretende analizar las producciones y uso de la lengua ayöök (mixe alto del norte) en espacios escolares y comunitarios en una comunidad rural dentro de un modelo educativo indígena alternativo: la Secundaria Comunitaria Indígena (SCI).

\section{LA ESCRITURA DEL MIXE}

La región mixe se encuentra ubicada en la sierra norte del estado de Oaxaca; está conformada por 19 municipios. La región se divide geográficamente en tres

3 En México las Escuelas Normales son instituciones de educación superior de formación para profesores de educación básica o fundamental. 
zonas: parte baja, media y alta. Esta misma división se ha utilizado para diferenciar las variantes lingüísticas del mixe. Para el INALI el mixe tiene 6 variantes: ayöök (mixe alto norte), ayuujk (mixe alto del centro), ayuujk (mixe alto del sur), ayuuk (mixe medio del este), eyuk (mixe medio del oeste) y ayuk (mixe bajo) (INALI, 2008).

En la década de 1960 el Instituto Lingüístico de Verano produjo varios diccionarios y traducciones bíblicas en las diferentes variantes de la región (SUSLAK, 2003). Sin embargo, la idea de un alfabeto unificado de toda la región mixe comenzó a formularse dentro de un proceso político de unificación regional. A fines de 1979, en la comunidad de Totontepec se realizó una reunión de autoridades Comunitarias y de Bienes Comunales de la región con el fin de compartir los problemas que tenía cada comunidad con respecto a la defensa de sus recursos naturales y el tema educativo ocupó importante espacio (MARTíNEZ, 2012). En esa reunión se firmó el "Manifiesto al Pueblo Mixe", donde se hacía una misma plataforma de lucha. Los primeros puntos referían al asunto educativo y a la escritura del mixe: "la educación que se proporcione a nuestros hijos debe desarrollarse doblemente, por un lado, está bien que aprenda hablar, leer y escribir español, siempre y cuando se vea la manera de lograr lo mismo con nuestro idioma mixe" (ROBLES; CARDOSO, 2007, p. 405).

En ese mismo año, se conformó con las autoridades de la región mixe alta el Comité de Desarrollo de los Recursos Naturales, Humanos y Culturales Mixes (CODREMI) cuyo objetivo fue construir una defensa colectiva contra las amenazas del gobierno, de grupos misioneros y de grandes intereses mineros y forestales. Un personaje importante en la organización fue Floriberto Díaz, originario de Tlahuitoltepec, quien comenzó a construir una perspectiva teórica para comprender la vida mixe, y que se denominó la comunalidad. La CODREMI impulsó el trabajo de la unificación de un alfabeto mixe a través de la Semana de Vida y Lengua Mixe. Entre 1983 y 1985 se realizaron seis reuniones con el fin de conseguir estandarizar la lengua mixe, de tal forma que cualquier variante pudiera ser escrita con las grafías contenidas en él (MARTínEZ, 2012). Esta actividad se sigue realizando, y tiene la finalidad de hacer talleres y pláticas para promover la escritura del mixe; en 2019 se realizó en la comunidad de San Marcos Moctum y en 2020 debido a la pandemia de Covid-19 se realizó de forma virtual.

Este ejercicio de unificación, impulsado por un grupo de líderes y lingüistas mixes, optó por construir un abecedario que respondía en gran medida a las 
propias características fonológicas del mixe sin mucha interferencia de la escritura del español durante el proceso de fijar las grafías (AGUILAR, 2014). A este abecedario se le ha llamado petaka en contra parte de otro alfabeto que existía en la zona baja mixe llamado bodega. En este nuevo alfabeto se considera que las grafías b, d y g, usados en español, ya no se escriban porque consideran que los sonidos /b/, /d/ y /g/ son alófonos de los fonemas /p/, /t/ y /k/ (en ese orden) (SANTOS, 2009, p. 29).

Siguiendo con este esfuerzo, el lingüista mixe Juan Carlos Reyes en 2005 publicó un manual que ha ayudado a la socialización del nuevo sistema de escritura y ha sido utilizado por los maestros de la región. En él se identifica que el alfabeto de la lengua ayuuk (variante del mixe medio del este) está conformado por las siguientes grafías: a, ä, e, ë, i, ü, j, k, l, m, n, o, ö, p, r, s, t, u, w, x, y, agregando la consonante llamada "glotal" representada por una apostrofe:' (REYES, 2005, p. 24). El número de vocales varía en cada variante, en la zona baja usan seis y en la variante de Totontepec hay nueve, pero siempre se representan con algunas de las grafías de este alfabeto.

Sin embargo, la estandarización de la escritura de lengua mixe no se ha logrado del todo. Esto puede deberse a varias razones. El antropólogo y lingüista Daniel Suslak menciona que se debe a que no se ha considerado "la variación fonética que tiene como resultado la falta de inteligibilidad entre las variantes del mixe" (2003, p. 559). El autor plantea que no son variantes de una misma lengua, sino que son lenguas diferentes provenientes de una misma familia lingüística (SUSLAK, 2003. Otra razón es que la unidad política como pueblo mixe, y por ende el discurso de unidad, ha sido impulsado y encabezado por un liderazgo político proveniente de la zona alta mixe y el abecedario fue impulsado desde esa zona. A esto se suma que los libros de texto en lengua indígena de educación primaria que distribuye la Secretaría de Educación Pública (SEP) a las escuelas están hechos en la variante del municipio de Guichicovi (mixe bajo) y del mixe medio (regiones con mayor población), lo cual ha agravado el proyecto de un abecedario común.

En este contexto de diversidad se han construido otras formas locales de escritura. En recientes años, la Asociación Civil Kumoontun ha comenzado a trabajar para socializar y promover la escritura del ayöök, variante lingüística de Totontepec de la región alta. Para ellos el abecedario del ayöök está conformado 
por las grafías consonantes: p, t, k, j, m, ts, n, w, x, y, y la glotal ('); y las nueve vocales: a, ä, e, ë, i, o, ö, u, ü.

Ante este panorama de encuentros y desencuentros para estandarizar la lengua, el presente texto analiza las producciones en lengua indígena que realizan los estudiantes de una Secundaria Comunitaria Indígena, y las reflexiones que ellos tienen acerca de su lengua ayöök y sus formas de representar conocimiento indígena. Esto en una comunidad de la zona mixe alta, con un territorio colindante con otras comunidades mixes y con comunidades zapotecas, con 750 habitantes aproximadamente, hablantes del ayöök como primera lengua.

\section{EL ALFABETO AYÖÖK EN LA SECUNDARIA COMUNITARIA}

Una de las innovaciones pedagógicas del modelo de las Secundarias Comunitarias Indígenas ${ }^{4}(\mathrm{SCl})$ es que la decisión de qué se estudia en la escuela está en manos de la comunidad ${ }^{5}$, es decir, hay una autonomía real en cuanto a contenidos. Esto implica que se organiza el trabajo escolar en proyectos de aprendizaje en sustitución de la división en 10 o más asignaturas planteada en el plan de estudios nacional de secundaria (Briseño, 2018). A diferencia de los proyectos que caracterizan a otros modelos educativos alternativos, los proyectos de aprendizaje de las $\mathrm{SCl}$ tienen la intención de durar todo un ciclo escolar, o si es necesario más tiempo, y son la base para organizar y vincular los conocimientos de varias disciplinas y el comunitario. El tema del proyecto es elegido en una Asamblea con la comunidad donde están invitados a participar los padres y madres de los estudiantes, las autoridades de la comunidad y de la secundaria. En esta Asamblea, los participantes vierten el tema que les interesa que sus hijos estudien en la Secundaria Comunitaria. En el tiempo del trabajo de campo, la mayoría de las mujeres madres no hablaban español o lo comprendían poco, por lo que toda la reunión se hacía en ayöök y los estudiantes hacen la mediación lingüística entre sus padres y madres y los maestros, si éstos no hablan la variante local.

4 Véase Briseño (2018), artículo en portugués sobre el modelo de las Secundarias Comunitarias Indígenas de Oaxaca.

5 En México existe un único programa educativo de educación básica o fundamental que se aplica en todas las escuelas indígenas y no indígenas. 
Al no tener una estructura disciplinar, cada secundaria comunitaria tiene tres educadores (indígenas y bilingües), uno por cada grado o grupo. El papel del maestro es guiar, orientar y crear estrategias para que los estudiantes sean sujetos activos y autónomos en la búsqueda de conocimientos y en la construcción de su aprendizaje (IEEPO, 2004). También se considera a las personas mayores de las comunidades como "portadores de conocimiento", ellos poseen el conocimiento comunitario y son a quienes se debe acudir para conocerlo. Esto se hace a través de las entrevistas, que son la herramienta fundamental para que los estudiantes investiguen el conocimiento que tienen las personas con más sabiduría en la comunidad (BRISEÑO, 2015). En la comunidad se les dice abuelitos o ap-ok, que da a entender el respeto hacia con ellos.

El trabajo etnográfico se realizó en la comunidad durante los años 20142015, observando de cerca las actividades escolares y acompañando a los estudiantes durante las entrevistas y otros eventos. A inicio del ciclo escolar 20142015, el grupo de 2o grado (8o de educación básica) de Secundaria Comunitaria Indígena investigó las características de la lengua mixe, en específico de la variante lingüística de la región, el ayöök. Este tema era parte del proyecto de aprendizaje diseñado por la generación desde el ciclo escolar anterior, llamado "Proceso histórico de la fundación y crecimiento de la comunidad". Algunas dimensiones que investigaron fueron: las variantes lingüísticas y las regiones donde se hablan, las diferencias entre ellas, el alfabeto del ayöök y "algunas palabras que ya no se dicen, sino que se usan en español". También produjeron textos en ayöök y reflexionaron sobre la importancia de escribir en la lengua indígena y no solamente en español. Estas actividades fueron guiadas por la maestra del grupo, Tania (todos los nombres usados son pseudónimos), originaria de la región, hablante del ayöök y con conocimiento lingüístico del mixe y sus variantes (de los maestros que pude observar fue la única que era hablante del ayöök, aunque otras hablaban otras variantes del mixe).

En octubre de ese año, los estudiantes de 2 o grado presentaron su trabajo de investigación sobre la lengua ayöök al grupo de 3 으 grado (9o de educación básica). Los estudiantes de 20 se dividieron los temas a presentar y cada uno fue explicado en ayöök y después en español. Primero hablaron sobre la importancia de escribir y hablar el mixe, mencionaron la palabra de los abuelos y los consejos que les dijeron "[...] nos distingue como persona indígena porque es la herencia 
de nuestros antepasados y es el más original de todos los pueblos, también cómo lo podemos conservarlo, hablándolo con los niños, no avergonzarse, escribiendo y leyéndolo" (Diario de campo). Después, presentaron las características de las seis variantes lingüísticas del mixe, situándolas geográficamente y describiendo los sonidos que las diferencian, en específico la cantidad de vocales en cada variante. Se enfocaron en la variante que ellos hablan, que es catalogada como mixe alto del norte.

Al terminar, dos estudiantes presentaron el alfabeto del ayöök, distinguiendo cada uno de los sonidos y las grafías que se utilizan. Construyeron un material donde se presenta cada grafía y una palabra como ejemplo y el dibujo para ayudar al a comprensión. En la Figura 1 se ve el material y el momento en el que uno de los estudiantes exponía esta información. Cada que se presentaba una grafía y su sonido, los estudiantes que estaban escuchando repetían la letra. Pareciera que estuvieran identificando ese sonido en las palabras que usan.

Figura 1 - Alfabeto ayöök

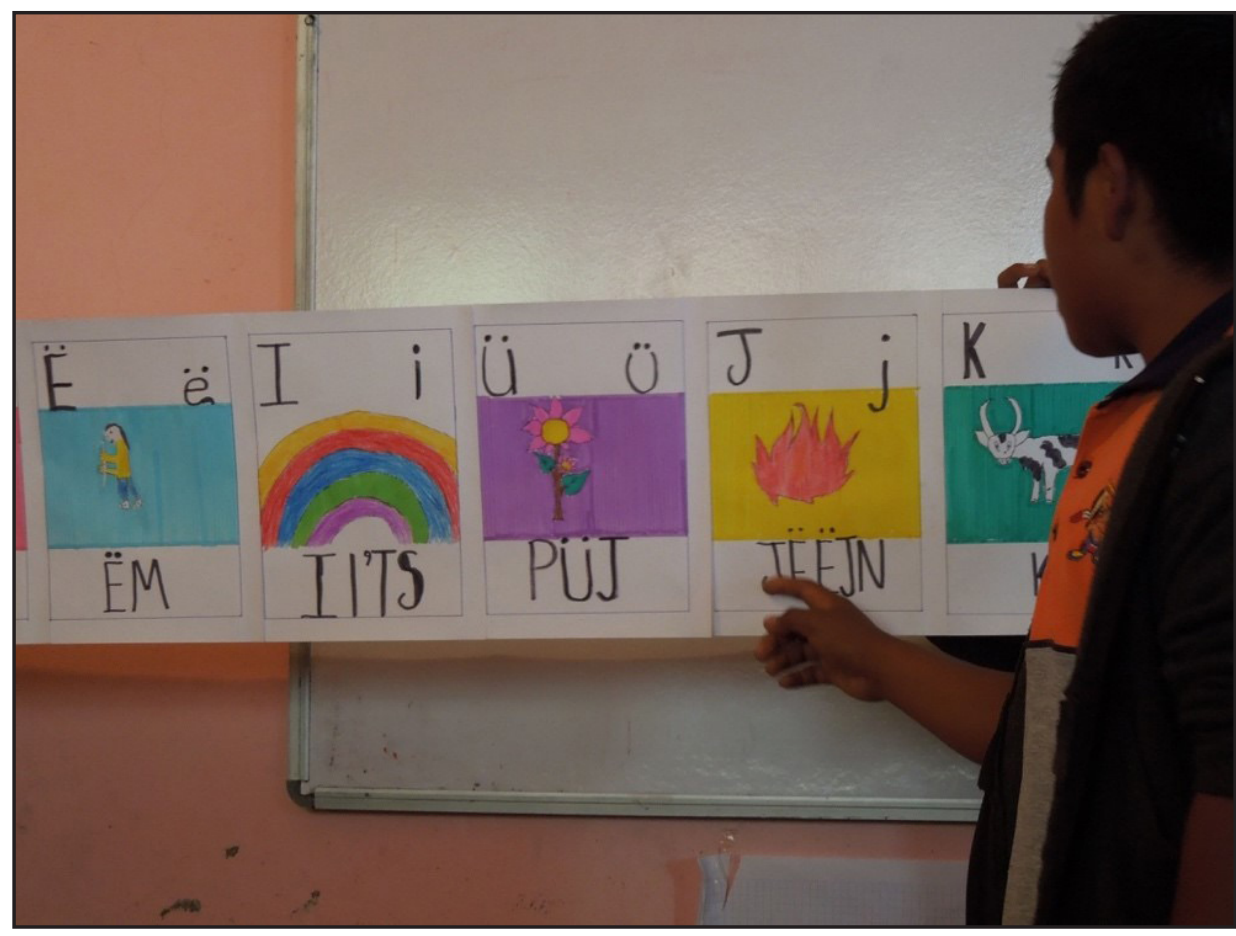

Fuente: Archivo personal. 
Después de explicar el alfabeto, los mismos dos estudiantes presentaron las grafías en español que no se usan para escribir en mixe, la b, la d y la g, señalaron las grafías del mixe usadas para presentar esos sonidos: /p/, /t/ y /k/. Es decir, los estudiantes utilizaron lo que se ha llamado el alfabeto petaka (anteriormente descrito). Con esto explicaron qué grafías no se deben usar al escribir en ayöök.

Por último, en esta sesión otros estudiantes presentaron algunos textos que escribieron en mixe sin ser traducción de otro texto en español: unas cartas de amor y una historia con moraleja. Al finalizar las maestras preguntaron si había dudas y una estudiante mencionó que a ella le habían enseñado que el sonido /b/ se escribía con la grafía w. La maestra Tania le contestó que lo importante por ahora era practicar el mixe escrito: "La idea es que, aunque no escribamos, así como dice el alfabeto siempre estemos escribiendo, si tenemos que hacer texto lo hagamos en mixe, primero en mixe y después en español" (Notas de campo, 17 octubre, 2014). Ante esto los estudiantes reaccionaron y comenzaron a hablar en mixe, uno de ellos comentó la importancia que tiene para la Secundaria Indígena que los maestros hablen mixe.

Este ejemplo muestra las reflexiones que hacen los estudiantes sobre la lengua mixe. Alejandra Pellicer con base en su estudio con niños mayas plantea que "la presencia simultánea de dos lenguas genera posibilidades de reflexión lingüística sobre las unidades de la lengua oral” (PELLICER, 2004, p. 268). En este caso, esta situación permite a los estudiantes hacer un análisis metalingüístico del ayöök, identificar los sonidos vocálicos, sus grafías y las características fonéticas, e incluso comentar sobre diferentes posibles letras que se han usado para escribir los sonidos.

Para describir las prácticas recurrentes de uso del ayöök y la producción de textos en lengua indígena y su relación con el español, presentamos la actividad de la entrevista que es un elemento fundamental dentro de la organización pedagógica de las Secundarias Comunitarias.

\section{CADENA DE LENGUAS ESPAÑOL-AYÖÖK EN LA PRÁCTICA}

La entrevista es un proceso que contiene diferentes actividades que se llevan a cabo tanto en español como en ayöök, en sus formas oral y escrita. A través de las observaciones de varios maestros y maestras en la $\mathrm{SCl}$, las formas en que se 
trabaja la entrevista son diferentes, pero para todos es el centro de la actividad de los proyectos de aprendizaje. Para los fines de este texto, tomaremos un caso para explicar la cadena de actividades y producciones que caracteriza a las actividades implicadas en la entrevista y su seguimiento en el aula. Se trata del trabajo que hizo la maestra Diana y su grupo de 12 estudiantes sobre el tema: la concepción del agua en la cultura mixe.

La primera actividad de la entrevista es construir un guion de entrevista. La dinámica utilizada por la maestra Diana fue que cada equipo (tres equipos) escribiera preguntas en español que querían hacerles a los abuelitos sobre el tema de investigación. Después un estudiante pasó al pizarrón y los demás le dictaron sus preguntas. Al tener todas las preguntas la maestra fue guiando una depuración, se eliminaron las preguntas que se repetían o se reformulaban para que abarcaran varias preguntas y se tuvo un guion de entrevista colectivo. Al completar las preguntas, los estudiantes las escribieron en sus cuadernos.

Después, la maestra indicó que se hiciera la traducción al mixe de cada una. Esta tarea no era sencilla para todos los estudiantes, sin embargo, al ser su segundo año en la $\mathrm{SCl}$, sus conocimientos de su lengua indígena escrita eran más amplios que cuando comenzaron la secundaria. En el trabajo de campo se observó cómo los estudiantes al ingresar a la secundaria no tenían un conocimiento homogéneo, ya que las prácticas escritas en ayöök son escasas tanto en la escuela primaria como en la vida comunitaria.

En el caso de este guion, pude observar que en cada equipo había un estudiante que sabía más sobre cómo escribir en ayöök y era quien apoyaba a sus compañeros en esta actividad; cuando no sabían como traducir alguna palabra o frase le preguntaban a la maestra. Al finalizar, todos tenían las preguntas traducidas en sus cuadernos. En la Figura 2 se presenta una fotografía de un cuaderno, donde se encuentran las preguntas en español construidas por todos y después la traducción al ayöök. En la imagen se puede ver que las preguntas en español están en tinta y las de lengua indígena están en lápiz, lo que da la posibilidad de ser borrado. 
Figura 2 - Guion de entrevista en español y en ayöök

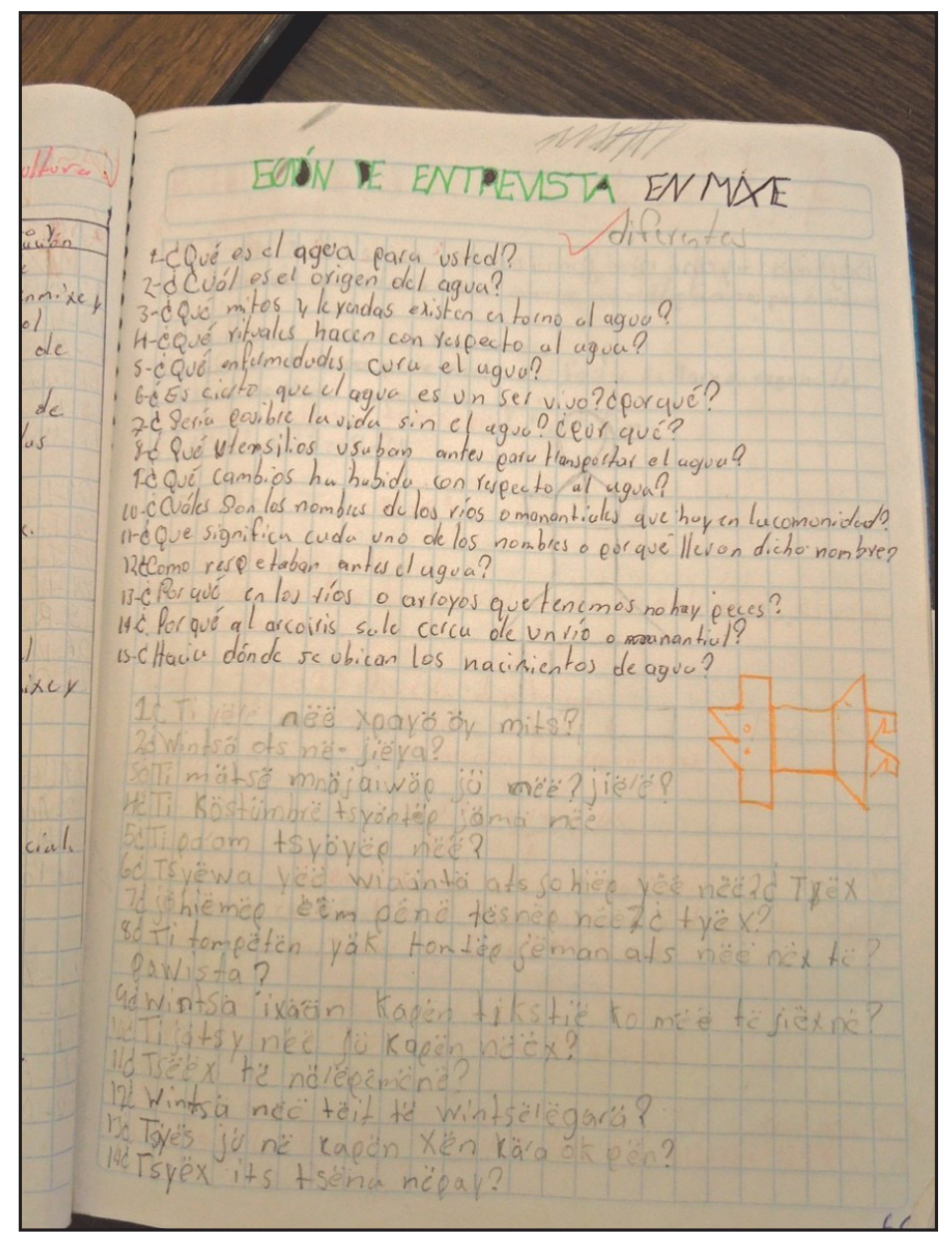

Fuente: Archivo personal.

Dentro del proceso de entrevista escolar comunitaria, al tener el guion de entrevista, se decide a qué abuelitos y abuelitas visitar. De las 16 entrevistas a las que tuve acceso, todas tuvieron las siguientes características: las preguntas al abuelito se hacían siempre en ayöök. Todos los estudiantes llevaban cuadernos, aquellos encargados en hacer las preguntas al portador o portadora lo consultaban y los estudiantes tomaban apuntes en sus cuadernos. Las respuestas de los abuelitos se hacían siempre en ayöök.

En el momento de hacer las preguntas, los estudiantes encargados veían sus cuadernos para recordar las preguntas, ponían en marcha la herramienta 
escrita. Sin embargo, no podemos saber si veían la escritura de español o la de ayöök. Esto significa que al estar haciendo las preguntas se iban guiando de lo escrito en el salón de clases y posiblemente haciendo una traducción simultánea al estar leyendo el español, o bien consultando la que estaba escrita en mixe. No utilizaban el guion como una estructura fija, sino que se iban acordando de los temas. Tanto es así que varias veces, al salir de visitar al abuelito, la maestra los regañaba por no hacer la pregunta correcta o por no avanzar en el guion que habían construido.

Durante la entrevista también sucedía otro proceso importante. Los estudiantes que no estuvieran encargados en hacer las preguntas tenían que escribir en sus cuadernos lo que el abuelito iba contando. Al revisar algunos de estos cuadernos, se vio que todos los apuntes sobre las respuestas en las entrevistas de los estudiantes estaban en español, lo que significa que ellos escuchaban en ayöök y en ese mismo momento apuntaban en español lo que decía el portador. Los estudiantes hacían una traducción simultánea ayöök-español al estar escuchando a los portadores. Esta capacidad de los niños de ser traductores ha sido ampliamente estudiada en contexto bilingües (ORELLANA, 2015; ORELLANA; REYNOLDS; DORNER; MEZAET, 2003). Sin embargo, lo importante es que los niños participan activamente en diferentes eventos de habla con adultos en diferentes contextos. Este papel de mediadores ampliaba las oportunidades para desarrollar empatía a otras personas y a sus experiencias (GUAN; GREENFIELD; ORELLANA, 2014, p. 332).

La cadena de producción en lengua ayöök atraviesa todo el proyecto de investigación del grupo. Con la información recabada de varias entrevistas, los estudiantes redactan un texto llamado "reporte de entrevista", en el cual explican lo que varios abuelitos dijeron sobre el subtema de investigación. Al tenerlo escrito en español lo vuelven a traducir a un ayöök escrito. Como bien dice la maestra Tania, no importa que la gramática y el vocabulario sean correctos, la intensión es que escriban y por tanto lean en su lengua. A su vez, los equipos transcriben estos reportes en computadora, tanto en español como en ayöök. Cada equipo transcribe el proyecto completo: los guiones de entrevista, los reportes de entrevista y otros textos redactados.

Las diversas formas de apropiación de los pueblos indígenas del español escrito muestran los diferentes usos que se fueron construyendo en situación de 
opresión y dominación, así como la relación con la lengua indígena escrita y con formas orales de trasmisión de conocimiento (ROCKWELL, 2006). Con base en estos planteamientos, uno de los conocimientos que se ha construido históricamente en el contexto multilingüe de la región de la sierra mixe es usar el español en la escritura y en comunicación con instancias del Estado, en particular las jurídicas y administrativas. Sin embargo, el modelo de Secundaria Comunitaria pretende generar situaciones en donde el mixe también se escriba directamente sin ser una traducción del español.

\section{PRODUCCIONES EN AYÖÖK}

El trabajo por proyectos de aprendizaje, basados en la investigación de situaciones y problemáticas locales, permite que los estudiantes tengan información de varias fuentes: de los abuelitos, de los libros, del internet, de sus familias, de ellos mismos y de la maestra. Cada proyecto está organizado en ámbitos o temas y a su vez dividido en subámbitos (subtemas). Con la información recabada los estudiantes deciden qué tipo de producción hacer para cada subámbito, por ejemplo: mapas, carteles, trípticos, líneas de tiempo, cuadros sinópticos, obras de teatro en ayöök, presentación de diapositivas utilizando un programa en la computadora, libros, periódicos murales, etc. Esta vasta producción de material es otra de las características fundamentales de la vida escolar de la Secundaria Comunitaria.

La mayoría de las producciones realizadas son textos escritos en español con alguna ejemplificación gráfica. Sin embargo, hay otro tipo de producciones que son representaciones gráficas por sí mismas, que no ilustran un texto escrito, sino que son en sí mismas textos. Elizabeth Boone llama esta forma de escritura como un sistema semasiográfico, en el cual el significado es dado por imágenes pictóricas y convencionales y por el contexto en el que participan (BOONE, 1994, p.18). Rossana Podesta (2007) estudió actividades con jóvenes nahuas en el centro de México, y mostró la importancia que tiene el dibujo para representar identidad, memoria-historia y territorio de una forma integral. Al hacer un paralelismo con los tres planos para estudiar la cultura escolar propuesto por Elsie Rockwell (2018), la escritura gráfica es una forma de representar un conocimiento que ha perdurado en las comunidades por siglos, al ser apropiada e incluso transformada por generaciones sucesivas. Es una práctica de larga duración que permea en la vida 
escolar de esta secundaria comunitaria, y remite a formas de conocer y de representar el conocimiento distintas a las planteadas por la cultura escrita alfabética.

Para mostrar cómo perdura esta forma de representar el conocimiento entre los mixes, utilizaremos un ejemplo de un Mapa del Territorio de la comunidad, realizado en 2017 por un estudiante del grupo de 1er año de la SCl. El proyecto de investigación se llamaba "El cultivo de hongos comestibles para su consumo y conservación" (véase Figura 3). El grupo realizó entrevistas para indagar la localización de los diferentes tipos de hongos en su territorio. Sin embargo, los estudiantes también tenían conocimientos sobre la fauna y flora de su comunidad, pues son hijos e hijas de campesinos que acompañan en varias de las tareas agrícolas a sus padres.

Figura 3 - Mapa del territorio y la localización de algunos hongos

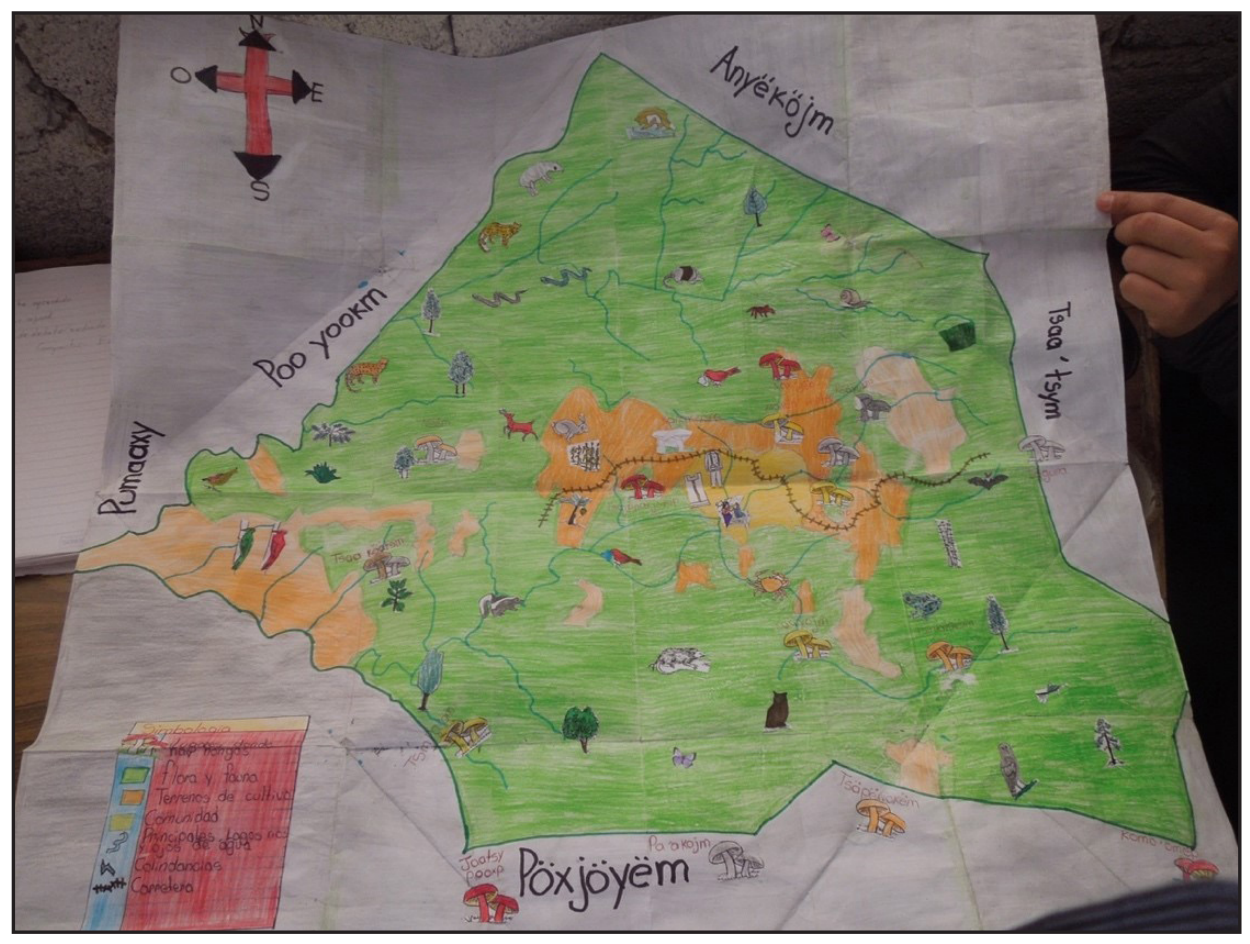

Fuente: Archivo personal.

En este mapa están representados algunos elementos del territorio: arroyos, animales, el asentamiento de la comunidad y los diferentes tipos de hongos. Los 
nombres de las comunidades con las que el territorio tiene colindancias están en mixe, así como los nombres de los hongos. Las comunidades de la región mixe siempre tienen dos nombres, uno en mixe y otro en español, sin ser traducción una de otra. En el mapa los nombres de los hongos están escritos por su nombre en el ayöök y se encuentran situados tanto afuera como dentro del territorio. Algunos son: pakjtsikë, küpë ëjkë, tsajpyawë, pa'oo'kën y jonteke puu'ts.

El mapa es resultado de información que los estudiantes obtuvieron de las entrevistas a abuelitos y con el comisariado de Bienes Comunales (autoridad comunitaria encargada de cuidar el territorio de la comunidad). Al reverso de esta producción, el autor del mapa escribió en español y en ayöök una presentación y descripción de su trabajo: "Este mapa está destinado a todos los habitantes...Pero nosotros no lo hicimos solos, sino que fuimos a entrevistar a los abuelos, también hicimos fichas y capturamos y buscamos en internet" (transcripción de la fotografía del mapa, archivo personal). En la figura 4 puede observarse el texto en las dos lenguas, así como las formas en las que acomodaron el texto con relación al mapa.

Figura 4 - Descripción escrita en ayöök y en español sobre el mapa de localización de hongos

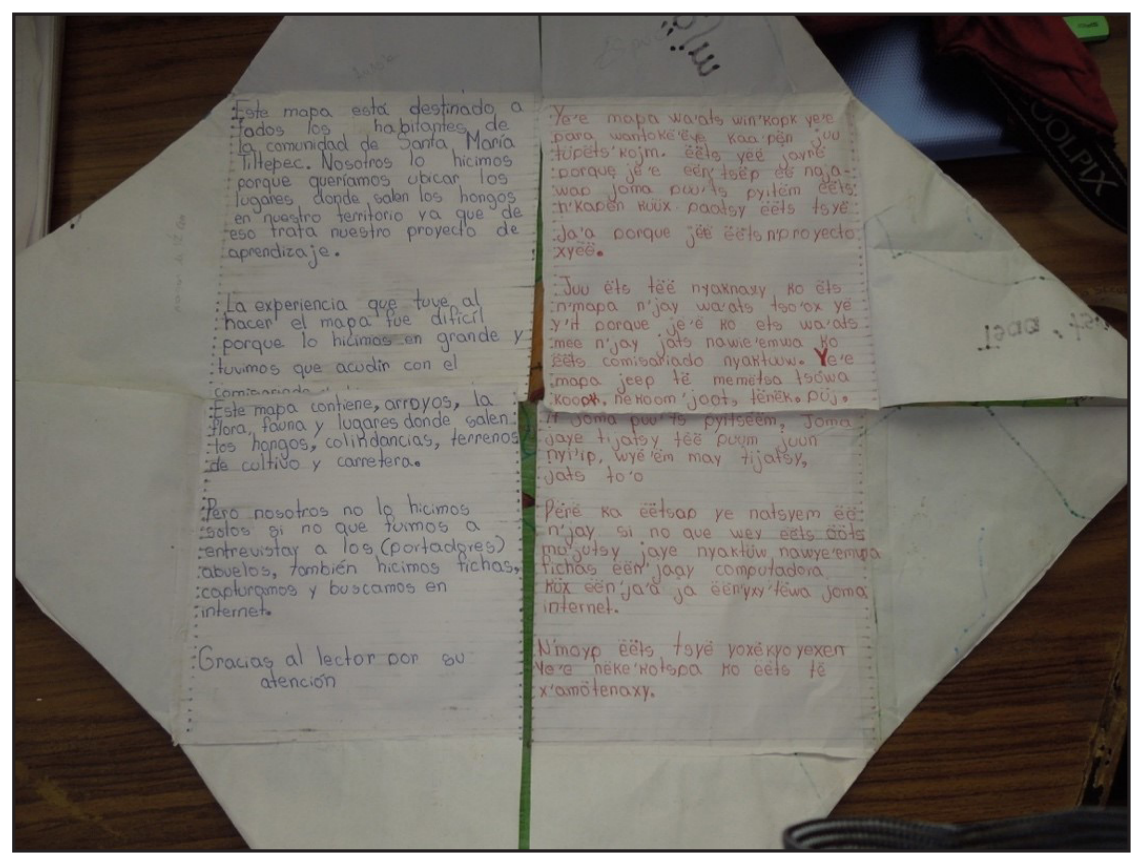

Fuente: Archivo personal. 
Esta producción muestra que el sesgo de la relación univoca entre lengua escrita y escolarización se rompe cuando hay flexibilidad de tiempos y de valorización de otros modos de representar lo que los estudiantes investigan y de expresar lo que aprenden. El efecto que tiene este tipo de producción es aumentar las formas comunicativas de los jóvenes y niños de representar sus conocimientos tanto en lengua indígena como en español y en otras formas de representación, como la gráfica.

La cantidad de producción gráfica en la vida cotidiana de la Secundaria Comunitaria es muy vasta y constante, es decir, no es producto de una clase de dibujo, es una práctica transversal gracias a la flexibilidad del modelo y la propuesta pedagógica. En la Figura 5 se presenta una imagen de una pared de un salón de clase, para recalcar la diversidad y amplia producción en español, ayöök y gráfica que se produce en la Secundaria Comunitaria. Es importante señalar que estas producciones no son estáticas y tienen funciones múltiples, pues se usan en presentaciones dentro de la clase, para la comunidad y en otros eventos fuera de la comunidad. La fotografía es un ejemplo representativo de lo producido al inicio del ciclo escolar y del ambiente que hay dentro de los salones de clases. Por cierto, es muy raro ver este tipo de exposición permanente de materiales en las paredes de las secundarias oficiales que siguen el programa por asignaturas, ya que, con el cambio constante de maestros en un grupo, no es permitido colocar nada que corresponda a una sola materia.

Figura 5 - Fotografía de un salón de clase

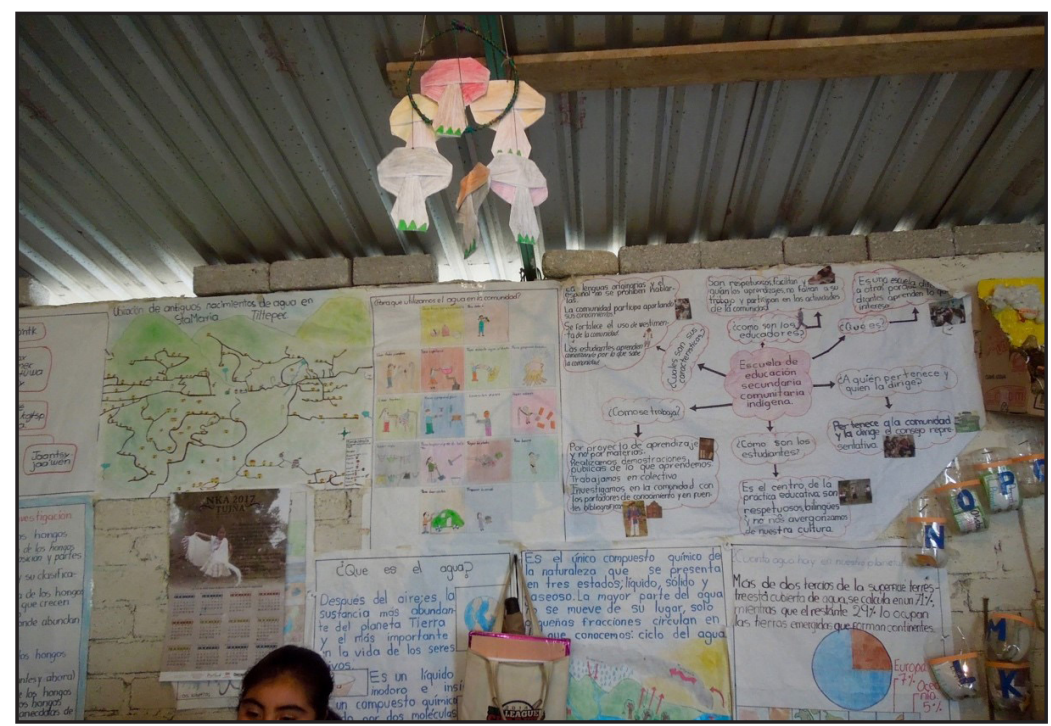

Fuente: Archivo personal. 
Estas representaciones de conocimientos se producen entre la apropiación de formas locales y la re-contextualización que cada estudiante o equipo le da, pues las transforman con su sello personal. Es decir, el conocimiento construido por los estudiantes en la experiencia de la Secundaria Comunitaria no es algo nuevo, pero tampoco es algo estático, no es un conocimiento indígena que está esperando a ser apropiado por los pobladores de ese pueblo indígena. Ese conocimiento de los estudiantes es el resultado de un proceso dialógico entre elementos socio-históricos comunitarios y la acción de sujetos con su historia y formas propias de mirarlo.

\section{CONCLUSIONES}

El entendimiento del uso y producción de textos en lengua indígena en situaciones escolares y su relación con el español debe de ser situada. La compleja dinámica entre las lenguas es única en cada lugar y mediada por diferentes factores. En este caso, el modelo pedagógico que plantea la Secundaria Comunitaria muestra la vitalidad del uso de las dos lenguas, español y ayöök, sin pautas preestablecidas y sin una en detrimento de la otra. En este sentido, los ejemplos aquí presentados muestran una forma de romper la relación colonial entre lenguas, es decir, el hecho de conocer y usar las dos en contextos y prácticas distintas posibilita a los jóvenes mixes de esta comunidad tener herramientas lingüísticas para salir de su comunidad como para quedarse dentro de ella. Propicia además una mayor reflexividad sobre las lenguas, su estructura y uso, así como sobre la traducción de una a otra.

Estas prácticas decoloniales entre lenguas se han planteado en varios modelos educativos bilingües e interculturales, pero el resultado observado en muchos casos es que el fortalecimiento de una lengua siempre va en detrimento de otra (MEEK; MESSING, 2007; LÓPEZ, 2006; SZULC, 2009). Lo que se muestra en la Secundarias Comunitarias, es que dadas las condiciones pedagógicas que fomenten el uso equilibrado, se puede fortalecer el uso, escrito y oral de las dos lenguas: ayöök y español.

La vitalidad del uso del ayöök no solo depende del modelo educativo, sino primero del uso de la lengua que de por sí existe en la comunidad; pero también se da porque dentro de ciertos momentos escolares se utilizan ciertas prácticas 
social-culturales. La entrevista con los abuelitos, que permite la apropiación de una relación de reciprocidad y respeto, obliga a un uso más equilibrado en el que se valora la lengua como vehículo de transmisión de conocimientos. La toma de decisiones sobre los temas a estudiar en forma de asamblea en donde las discusiones se hacen en la lengua indígena es otra instancia de uso equilibrado de ambas lenguas. Esta particularidad muestra la fortaleza de un modelo que no determina cierta hora y asignatura para la enseñanza y práctica de la lengua indígena sino a la libertad, flexibilidad y al uso espontaneo de la misma. Consideremos que el resultado va mas allá del mero uso de las lenguas, para generar una reflexión metalingüística tanto del español como del ayöök, así como sobre la organización curricular en las secundarias en general.

Al imaginar que esta Secundaria Comunitaria no estuviera en la comunidad, podríamos asegurar que los jóvenes, tanto si siguieran estudiando fuera de la comunidad o dejaran de estudiar, no tendrían los recursos lingüísticos para poder escribir ni español ni mixe. En contextos coloniales como México, el ser bilingüe y comprender el funcionamiento de las lenguas posibilita una resistencia fundamental para la valoración de su propia lengua, sus usos y fortaleza identitaria.

\section{REFERENCIAS}

AGUILAR, Yasnaya. ¿Construyendo un abecedario para una lengua indígena? Algunas ideas a considerar. El País, enero, 2014. Disponible: http://archivo.estepais.com/site/2014/ construyendo-un-abecedario-para-una-lengua-indigena-algunas-ideas-a-considerar/

BOONE, Elizabeth Hill. Writing and recording knowledge. In: BOONE, Elizabeth; MIGNOLO, Walter (Eds.). Writing without words: alternatives literacies in Mesoamerica and the Andes. Durkham: Duke University Press, 1994. p. 3-26.

BRISEÑO, Julieta. Os jovens, o pensamento-outro e a micro-geopolítica do conhecimento integeracional nas Escolas Secundárias Comunitárias Indígenas de Oaxaca, México. Arquivos Analíticos de Políticas Educativas, v. 26, n. 88, 2018. Disponible: https://doi. org/10.14507/epaa.26.3505

BRISEÑO, Julieta. Entrevistas comunitarias, la recuperación del conocimiento comunitario desde la escuela. El caso de las Secundarias Comunitarias Indígenas, Oaxaca, México. Archivos Analíticos de Políticas Educativas, v. 23, n. 95, 2015. Disponible: http://dx.doi. org/10.14507/epaa.v23.2081 
COLLINS, James; BLOT, Richard. Literacy and Literacies: texts, power and identity. Cambridge: Cambridge University Press, 2003.

CIFUENTES, Bárbara; MOCTEZUMA, Jose Luis. The Mexican indigenous languages and the national censuses: 1970-2000. In: HIDALGO, Margarita (Comp.). Mexican Indigenous Languages at the Dawn of the Twenty-First Century. Berlin: Walter de Gruyter, 2006. p. 191-248.

DE LEON, Lourdes. Medialetics in the creation of Mayan peer cultures. In: BELL, Joshua; KUIPERS, Joel (Ed). Linguistics and Material Intimacies of Cell Phones. New York: Routledge, 2018. p. 101-27.

FLORES F., Jose Antonio. Los rostros del español en el náhuatl ayer y hoy. Estudios de Cultura Nahuatl, manuscrito, 2020.

GUAN, Shu-Sha; GREENFIELD, Patricia; ORELLANA, Marjorie. Translating into understand: language brokering and prosocial development in emerging adults from immigrant families. Journal of Adolescent Research, v. 29, n. 3, p. 331-55, 2014.

HAMEL, Rainer; BALTAZAR, Ana; MARTINEZ, Betzabé. La construcción de la identidad P'urhepecha a partir de la educación intercultural bilingüe propia p'urhepecha. Trabalhos em Lingüistica Aplicada, v. 57, n. 3, p. 1377-412, sept./dez. 2018. Disponible: http:// dx.doi.org/10.1590/010318138653739444541

INSTITUTO ESTATAL DE EDUCACIÓN PÚBLICA DE OAXACA. Proyecto de secundarias para la atención de comunidades pertenecientes a los pueblos originarios. Documento de trabajo, 2004.

INSTITUTO NACIONAL DE LENGUAS INDÍGENAS. Catálogo de las Lenguas Indígenas Nacionales: variantes lingüísticas de México con sus autodenominaciones y referencias geoestadísticas. México, 2008. Disponible: https://www.inali.gob.mx/clin-inali/

LÓPEZ, Luis. Desde arriba y desde abajo: Visiones contrapuestas de la educación intercultural bilingüe en América Latina. Quinasay - Revista de Educación Intercultural Bilingüe, v. 4, n. 4, p. 81-100, 2006.

MARTÍNEZ, Daniel. Las organizaciones comunitarias y sus propuestas de educación, lingüística y cultural de la región Ayuujk. Ciudad de México, UPN, 2012. 140 p. Tesis (Doctorado en Sociología de Educación) - Universidad Pedagógica Nacional, Ciudad de México, 2012.

McCARTY, Teresa. Revitalising Indigenous Languages in Homogenizing Times. Comparative Education, v. 39, n. 2, p. 147-63, 2003. 
MEEK, Barba; MESSING, Jaqueline. Framing Indigenous Languages as Secondary to Matrix Languages. Anthropology \& Education Quarterly, v. 38, n. 2, p. 99-118, 2007.

MEYER, Louise. Hacia una alternativa de formación indígena bilingüe e intercultural: una pedagogía liberadora desde la "comunalidad". Revista Latinoamericana de Educación Inclusiva, v. 4, n. 1, p. 83-103, 2010.

ORELLANA, Marjorie. Translanguaging within enactments of quotidian interpretermediated interactions. Journal of Linguistic Anthropology, v. 24, n. 3, p. 315-38, 2015.

ORELLANA, Marjorie; REYNOLDS, Jennifer; DORNER, Lisa; MEZA, María. In other words: translating or 'para-phrasing' as a family literacy practice in immigrant households. Reading Research Quarterly, v. 38, n. 1, p. 12-34, jan./mar. 2003.

PELLICER, Alejandra. Así escriben los niños mayas su lengua materna. Ciudad de México: Plaza y Valdés, 2004.

PELLICER, Dora. Linguistic Diversity in the Classroom and Beyond. Is it Wrong or Just Different? Indigenous Spanish in Mexico. Social Issues in Applied Linguistics, v. 18, n. 2, p. 189-98, 2010.

PODESTA, Rossana. Encuentro de Miradas, el territorio visto por diversos autores. México: SEP, 2007.

QUIJANO, Aníbal. Cuestiones y Horizontes, Antología Esencial. Buenos Aires: Clacso, 2014.

REYES, Juan Carlos. Aportes al proceso de enseñanza aprendizaje de la lectura y la escritura de la lengua ayuuk. Primera edición. Oaxaca: CEA-UIIA, 2005.

ROBLES, Sofia: CARDOSO, Rafael (Comp.). Floriberto Díaz. Escritos. México: UNAM, 2007.

ROCKWELL, Elsie. Tres planos para el estudio de las culturas escolares. En ROCKWELL, E. Vivir entre escuelas. Relatos y presencias. Antología esencial. Buenos Aires: CLACSO, 2018. p. 173-188.

ROCKWELL, Elsie. Apropiaciones indígenas de la escritura en tres dominios: Religión, Gobierno y Escuela. Cultura Escrita \& Sociedad, n. 3, pp 161-218, 2006.

SANTOS, María del Rosario. Alfabetización en lengua ayuuk (mixe) por transferencia de competencias, Ciudad de México, UPN. 2009. 152 p. Tesis (Doctorado en Educación Indígena) - Universidad Pedagógica Nacional, Ciudad de México, Mexico, 2009. 
SUSLAK, Daniel. The story of Ö: orthography and cultural politics in the Mixe highlands. Pragmatics, v. 13, n. 4, p. 551-63, 2003.

SZULC, Andrea. Becoming Neuquino in Mapuzugun: Teaching Mapuche Language and Culture in the Province of Neuquén, Argentina. Antrophology \& Education Quarterly, v. 40, n. 2, p. 129-49, July 2009.

\section{Sobre las autoras:}

Julieta Briseño-Roa: Antropóloga y Doctora en Ciencias en la especialidad en Investigaciones Educativas por el Departamento de Investigaciones Educativas (DIE), Cinvestav. Es profesora-investigadora en el Centro de Investigaciones y Estudios Superiores en Antropología Social (CIESAS) y profesora de asignatura en la UNAM. Sus temas de investigación son educación indígena, apropiaciones comunitarias de la escuela, prácticas escolares comunitarias, translingüismo y epistemologías indígenas con perspectiva decolonial. ProfesoraInvestigadora CIESAS, sede CDMX. E-mail: julietabriseno@ciesas.edu.mx, Orcid: https://orcid.org/0000-0003-0641-1567

Elsie Rockwell Richmond: Mexicana, historiadora y antropóloga de formación, optó por dedicarse al campo educativo. Cuarenta y cinco años como investigadora en el Centro de Investigación y de Estudios Avanzados, Cinvestav, le han permitido explorar una diversidad de lenguas, palabras y culturas en México y otros países. La Antología, Vivir entre escuelas (CLACSO, 2018) reúne buena parte de sus publicaciones. En 2019 recibió el Doctorado Honoris Causa de la Universidad Nacional de Córdoba. Profesora Emérita, DIE-Cinvestav, Mx. E-mail: elsierockwell@gmail.com, Orcid: https://orcid.org/0000-0002-3981-2108

Recibido el: 20 de mayo de 2020.

Aprobado para publicación el: 20 de noviembre de 2020. 